(C) 2018 IEEE. Personal use of this material is permitted. Permission from IEEE must be obtained for all other uses, in any current or future media, including reprinting/republishing this material for advertising or promotional purposes, creating new collective works, for resale or redistribution to servers or lists, or reuse of any copyrighted component of this work in other work.

\title{
CROP TYPE MAPPING BASED ON SENTINEL-1 BACKSCATTER TIME SERIES
}

\author{
M. Arias; M.A. Campo-Bescós; J. Álvarez-Mozos
}

Public University of Navarre, Department of Projects and Rural Engineering, Arrosadia Campus 31006 Pamplona. Correspondence: jesus.alvarez@unavarra.es; Tel.: +34-948-169235

\begin{abstract}
The high revisit time of Sentinel-1 (S1) observations enables the design of crop type mapping approaches exploiting the backscatter time series observed for the different crops. The objective of this study is to propose a supervised crop classification methodology based on the temporal signature of crops. With this aim 29 dual-pol S1 observations acquired over an agricultural area of Spain, where ground truth was available, were processed. The classification approach was based on the temporal signatures obtained for each polarization channel (VH, VV and the cross-pol ratio) for the different crops. Highest accuracies were obtained when fields were assigned to the class that minimized the RMSE, with an overall accuracy of $79 \%$ and best results for rapeseed, sunflower, alfalfa and barley.
\end{abstract}

Index Terms - crop type mapping, supervised classification, Sentinel-1, time series

\section{INTRODUCTION}

C-band backscatter data have proven to be useful for crop type mapping, as long as observations are available in key moments where phenological differences between crops are apparent [1]. Sentinel-1 (S1) mission's unprecedented revisit time [2], allows for a detailed temporal monitoring and holds great expectations for passing from approaches based on few observations and sophisticated classification algorithms to approaches where the potential of the time series information can be exploited with rather simple classification algorithms [3]. Besides, the default configuration over land areas, with $\mathrm{VH}+\mathrm{VV}$ dual-pol capabilities have been shown to be sensitive to capture differences between crops [4]. The objective of this work is to propose a supervised crop classification approach based on the temporal signature of backscatter at different polarizations for the different crops.

\section{MATERIALS AND METHODS}

\subsection{Study area}

The study area corresponds to the agricultural region of Pamplona (province of Navarre, Spain) (Fig. 1), an area of $778 \mathrm{~km}^{2}$ where rain-fed agriculture is the main land use, with winter cereals the most common crops. The climate is humid Mediterranean with precipitations over $700 \mathrm{~mm}$ per year.

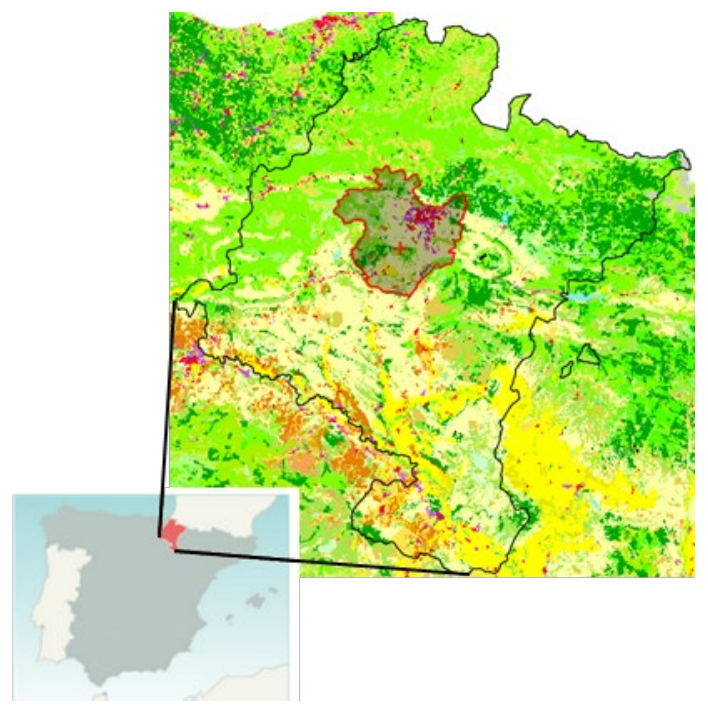


Figure 1. Study area located on the province of Navarre (Spain).

Field inspections were carried out from June to August 2016 as part of the verifications of the EU Common Agricultural Policy (CAP) subsidy declarations. Most crops in the area were rain-fed, except for corn. A $10 \mathrm{~m}$ buffer was applied to avoid mixed pixels, and only fields larger than 0.2 ha were considered, leaving a total of 600 fields. The number of fields available per crop type varied markedly and reflected the relative proportion of each crop in the region, being the most frequent winter cereals like wheat (188) and barley (145), followed by beans (51), rapeseed (47), peas (43), oats (41) and sunflower (31), and in a much lower proportion fallow (24), corn (17), alfalfa (7) and vetch (6).

\subsection{Sentinel-1 data}

Sentinel-1A (S1A) dual-pol (VH+VV) Interferometric Wide Swath (IW) mode data acquired over the study area from September 1, 2015 to September 1, 2016 were used. Only scenes obtained in ascending mode (relative orbit 103ASC) were used to avoid the influence of varying image geometry. A total of 29 scenes were used, which were downloaded as Ground Range Detected (GRD) products and processed to obtain backscatter coefficient values in the two available polarizations $\left(\sigma^{0} \mathrm{VH}\right.$ and $\left.\sigma^{0} \mathrm{vv}\right)$ and their ratio $\left(q=\sigma^{0} \mathrm{VH} / \sigma^{0} \mathrm{VV}\right)$ in $\mathrm{dB}$ units.

Scenes were processed using a processing graph built on Sentinel Application Platform (SNAP) and consisting of (1) orbit metadata refinement, (2) thermal noise removal, (3) calibration to $\beta^{0}$, (4) Gamma MAP $3 \times 3$ speckle filter, (5) Range Doppler terrain correction and (6) radiometric normalization of the topography using the local incidence angle to obtain $\sigma^{\circ}$. Finally the cross-polarization ratio $q$ was calculated and $\sigma^{0}$ and $q$ values were transformed to $\mathrm{dB}$.

\subsection{Time series extraction and classification}

Ground truth data was randomly divided into a training set $(2 / 3)$ and a test set $(1 / 3)$. With the training set backscatter time series were built for $\sigma^{0} \mathrm{VH}, \sigma^{0} \mathrm{Vv}$ and $q$ for each crop type, with the aim of obtaining a backscatter temporal signature representative of each crop. These temporal signature curves were then used as a reference for classification assigning each field to the crop type that maximized its agreement. To evaluate the agreement two different measures were used: the determination coefficient $\left(\mathrm{R}^{2}\right)$ and the root mean squared error (RMSE), on the three polarization channels available $\left(\sigma_{\mathrm{VH}}, \sigma^{0} \mathrm{Vv}\right.$ and $q$ ). Finally, a further option was explored that consisted of assigning the field to the crop that maximized the agreement on the three polarization channels globally, this option was called Global. Summing up, eight different classification models were evaluated by combining two assignation measures ( $\mathrm{R}^{2}$ and RMSE) and four classification methods ( $\sigma^{0} \mathrm{vH}, \sigma^{\circ} \mathrm{vv}, q$ and Global).
In order to evaluate the accuracy of the eight different classification models, the test set was classified and confusion matrices were extracted for each model. The Overall Accuracy (OA), i.e. the proportion of correctly classified validation fields, was computed for each model. Results were also evaluated per crop by computing their Producer's (PA) and User's Accuracy (UA). PA is defined as the proportion of correctly classified test fields for a given class, and UA as the proportion of fields assigned to a given class that actually corresponded to this class.

\section{RESULTS AND DISCUSSION}

\subsection{Backscatter time series}

The temporal signatures of the eleven different crop types considered revealed different backscattering behaviors along the growing season (Fig. 2 shows a selection of four crops). The two different polarization channels (VH and VV) showed mostly a strongly varying signature with no clear trends, whereas very remarkably the cross-pol ratio $(q)$ responded quite smoothly and monotonically to the growth of the different crops (Fig. 2). This might be explained by the fact that $\mathrm{VH}$ and VV channels were mostly sensitive to different phenological events and also to variations in soil surface conditions (moisture and roughness), whereas $q$ seemed to be more insensitive to these individual variations and only affected by the biomass development of crops.

Winter cereals (wheat, barley and oats) showed a decreasing trend in $\mathrm{VV}$ during the period of vegetative growth, caused by the attenuation of surface scattering due to the vertical stems of plants. Rapeseed and legume crops (peas, beans and vetch) had an increasing trend in VH due to their bushy structure that contributed to volume scattering. Summer crops (sunflower and corn), had strongly varying $\mathrm{VH}$ and $\mathrm{VV}$ responses reflecting soil moisture dynamics and roughness variations due to tillage until sowing in May ( $\sim 250$ day).

\subsection{Classification results}

OA results (Table 1) show that VV polarization provided a slightly higher accuracy than VH. Remarkably, classification based on $q$ temporal signature lead to the poorest results. So, $q$ seemed to be sensitive to crop growth but in a similar way for the different crops studied, whereas $\mathrm{VV}$ and $\mathrm{VH}$ revealed phenological differences that were useful for classification. Assignation based on RMSE produced, in all cases, higher $\mathrm{OA}$ values than when based on $\mathrm{R}^{2}$. Best classification results were obtained with the Global classification with OA values up to $79 \%$.

Table 1. Overall Accuracy (OA) of classifications based on $\mathrm{R}^{2}$ or RMSE assignation for the different polarizations

\begin{tabular}{|l|l|l|l|l|}
\hline $\mathrm{OA}(\%)$ & $\sigma^{0} \mathrm{VH}$ & $\sigma^{0} \mathrm{VV}$ & $q$ & Global \\
\hline $\mathrm{R}^{2}$ & $67 \%$ & $72 \%$ & $58 \%$ & $77 \%$ \\
\hline
\end{tabular}




\begin{tabular}{|l|l|l|l|l|}
\hline RMSE & $72 \%$ & $73 \%$ & $60 \%$ & $79 \%$ \\
\hline
\end{tabular}

Looking in detail at the results per crop (Fig. 3) it can be observed that PA and UA values varied strongly from crop to crop, with poorest results for legume crops (peas, beans and vetch), that had very similar temporal signatures and were not correctly discriminated. Cereal crops had better results, being barley the best classified, while wheat and oats were confounded and obtained lower PA and UA values, respectively. Rapeseed and sunflower achieved the best results, with high PA and UA values $(>90 \%)$ when classification was based on VH or Global and assignation was done using RMSE. Corn had intermediate results independently of the assignation method used, with medium PA and high UA values and confusion with fallow fields. Probably, an extension of the time period considered for extracting the temporal signatures would avoid its confusion with fallow fields, as this would enable a complete monitoring of its growth cycle.
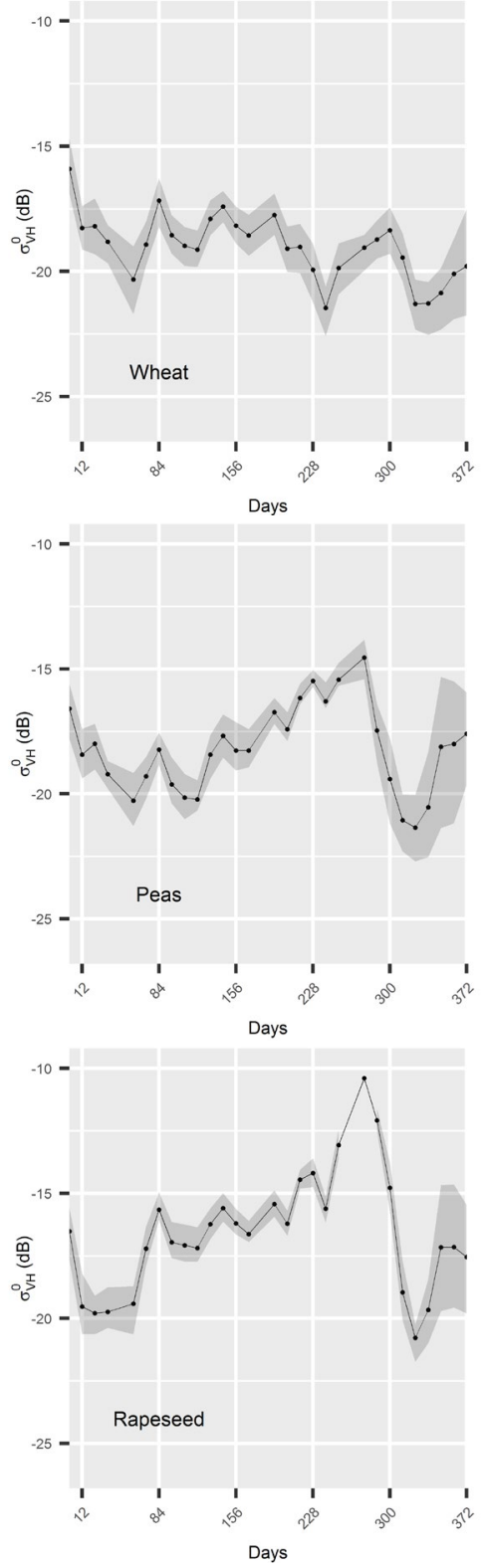
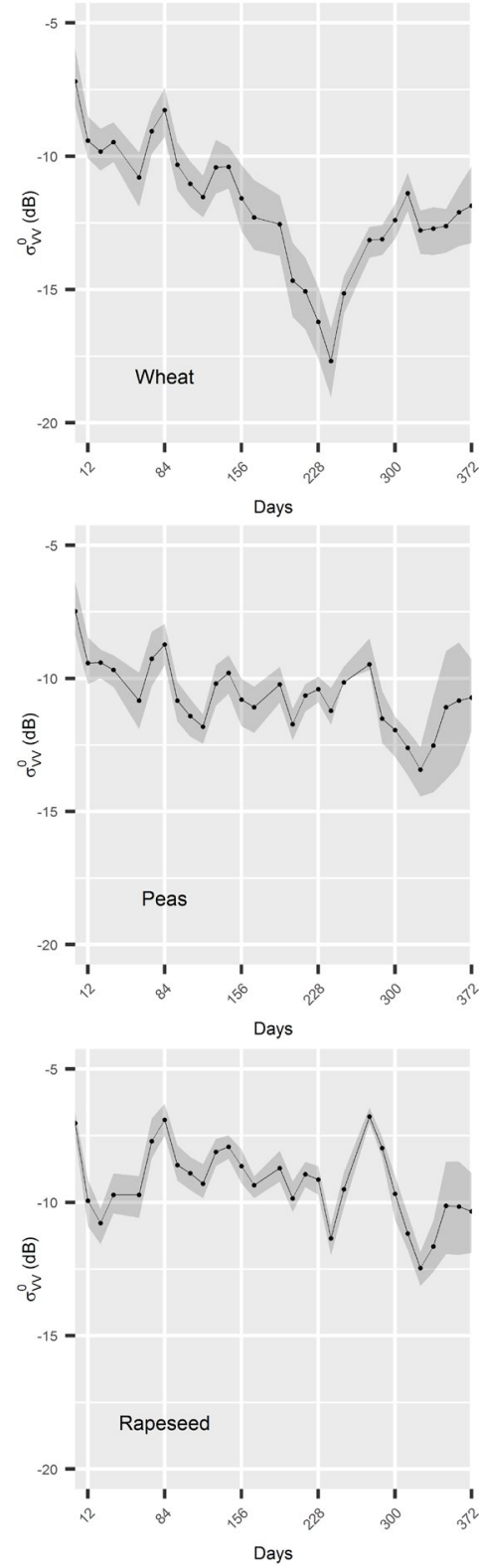
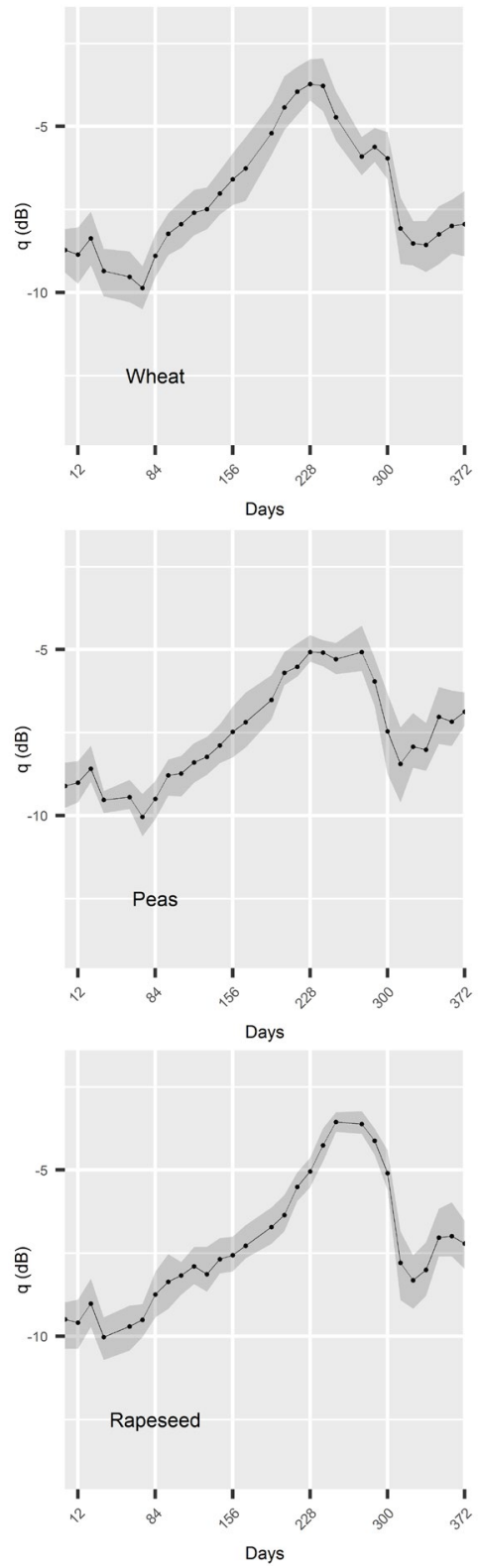

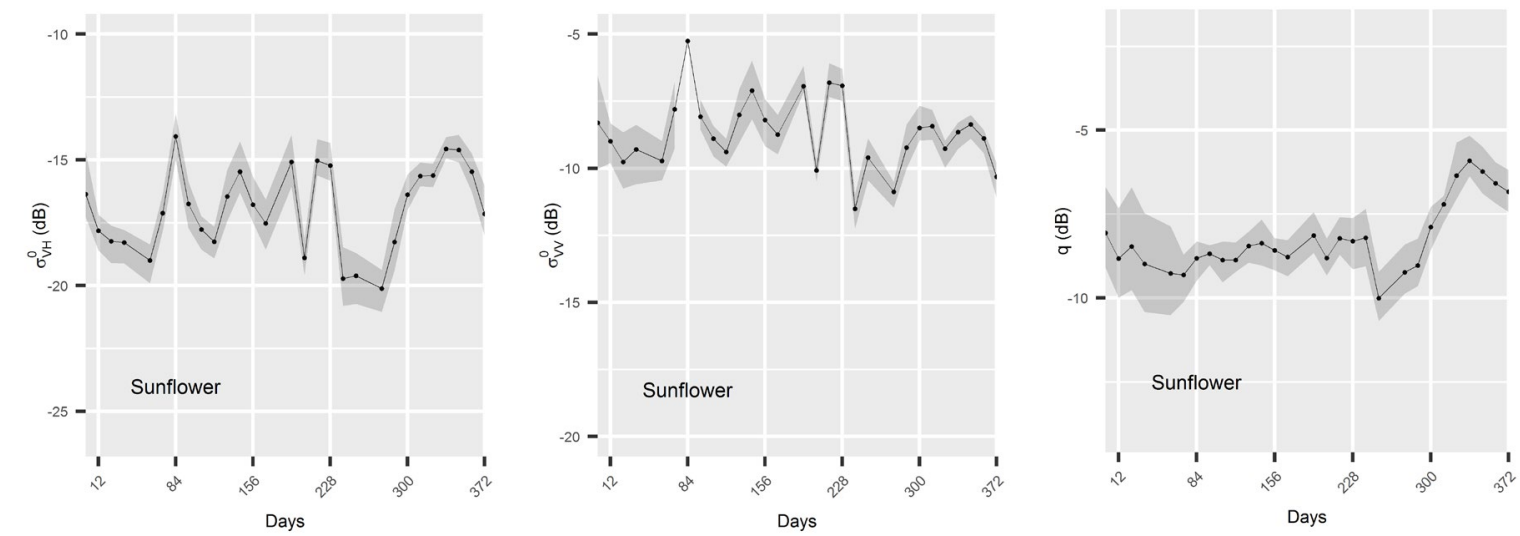

Figure 2. Temporal signatures of four representative crop types for the two polarizations studied: VH (left) and VV (middle) and cross-pol ratio $q$ (right). The black line represents the mean response and the grey area the region between the first and third quartile. Days start counting from September 1, 2015.
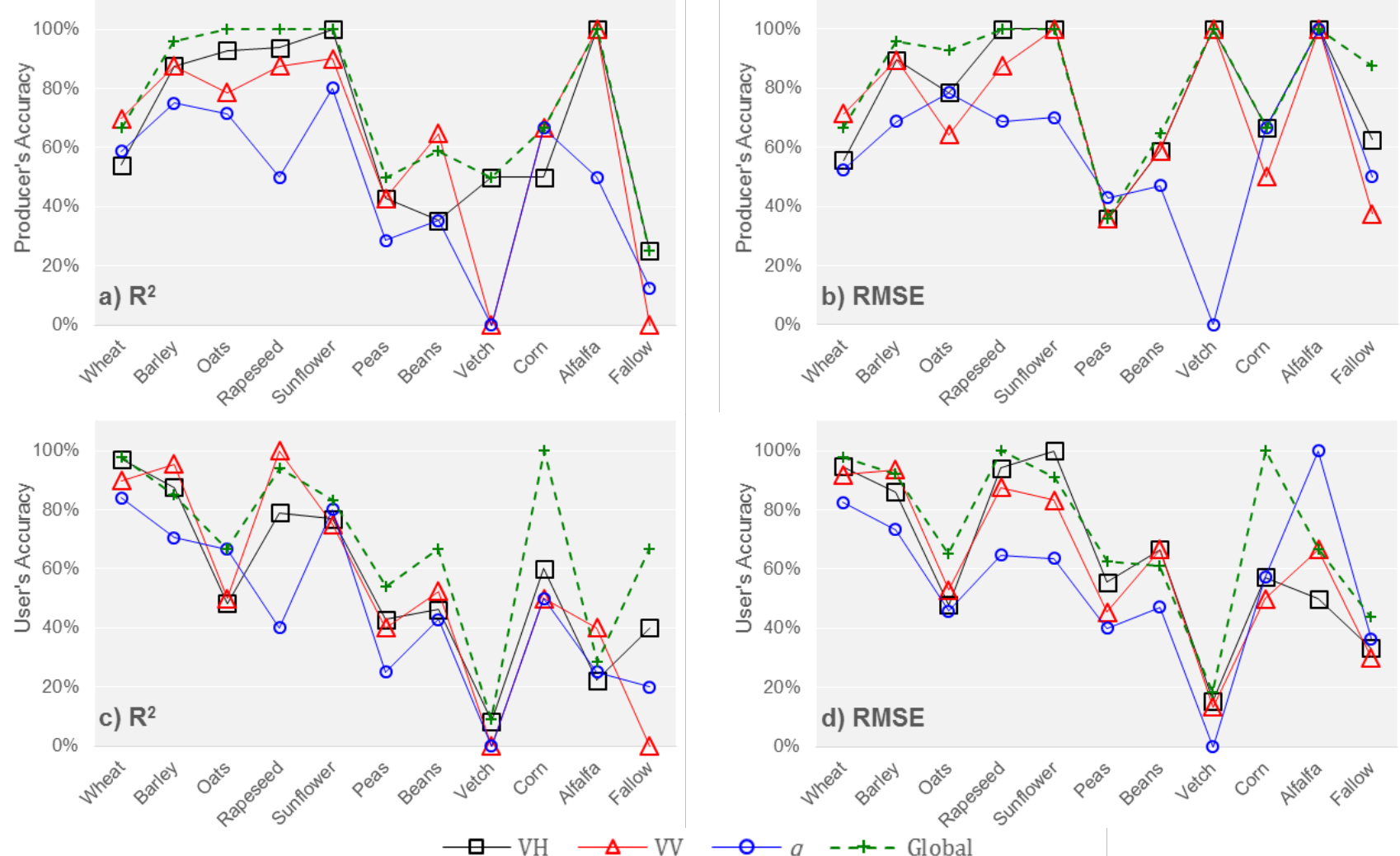

Figure 3. Producer's Accuracy ( $a$ and b) and User's Accuracy (c and d) results per crop for classifications based on different polarization channels (see legend) and assignation based on $\mathrm{R}^{2}$ ( $\mathrm{a}$ and $\mathrm{c}$ ) and RMSE ( $\mathrm{b}$ and d).

Alfalfa was the only crop that obtained the best results when classification was based on $q$ (and RMSE assignation). This could be due to its characteristic growth phase that was interrupted several times by the periodical cutting of the crop, leading to a serrated $q$ temporal signature. Finally, fallow fields were best identified using the Global classification and RMSE assignation, yet UA values were low since many different crops were incorrectly classified as fallow.

\section{CONCLUSION}

A supervised classification approach based on Sentinel-1 backscatter time series was proposed, which exploited the temporal signatures of crops. Temporal signatures of backscatter at VV and $\mathrm{VH}$ revealed different phenological events, whereas the cross-pol ratio $(q)$ appeared sensitive to crop growth. The agreement to the training temporal signatures was measured with $\mathrm{R}^{2}$ and RMSE, yet the latter yielded the highest classification accuracies. Best results 
were obtained when the classification algorithm optimized the match to the three temporal signatures (VH, VV and $q$ ), with an overall accuracy of $79 \%$. Results per crop varied, with the highest accuracies obtained for rapeseed, sunflower, alfalfa and barley, followed by wheat, oats and corn. Legume crops like peas, beans and vetch obtained the poorest results.

\section{ACKNOWLEDGEMENTS}

The authors thank the Government of Navarre for providing the ground truth data. This work was partly funded by project CGL2016-75217-R (MINECO/FEDER, EU) and project PyrenEOS EFA 048/15, the latter has been $65 \%$ cofinanced by the European Regional Development Fund (ERDF) through the Interreg V-A Spain-FranceAndorra programme (POCTEFA 2014-2020).

\section{REFERENCES}

[1] H. Skriver, "Crop classification by multitemporal Cand L-band single- and dual-polarization and fully polarimetric SAR," IEEE Trans. Geosci. Remote Sens., vol. 50, no. 6, pp. 2138-2149, Jun. 2012.

[2] R. Torres et al., "GMES Sentinel-1 Mission," Remote Sens. Environ., vol. 120 pp. 9-24, May 2012.

[3] T. Whelen and P. Siqueira, "Use of time-series L-band UAVSAR data for the classification of agricultural fields in the San Joaquin Valley," Remote Sens. Environ., vol. 193, pp. 216-224, May 2017.

[4] A. Larrañaga and J. Álvarez-Mozos, "On the Added Value of Quad-Pol Data in a Multi-Temporal Crop Classification Framework Based on RADARSAT-2 Imagery,” Remote Sens., vol. 8, no. 4, art. 335, Apr. 2016. 